\title{
Prognostic Impact of Copeptin and Mid-Regional Pro-Adrenomedullin in Chronic Heart Failure with Regard to Comorbidities
}

Spinarova $\mathbf{L}^{1}$, Spinarova $\mathbf{M}^{* 1}$, Goldbergova-Pavkova $\mathbf{M}^{2}$, Spinar $\mathrm{J}^{3,4}$, Parenica $\mathrm{J}^{3}$, Ludka $\mathbf{O}^{3,4}$, Labr $\mathbf{K}^{1}$, Malek $\mathrm{F}^{5}$, Ostadal $\mathbf{P}^{5}$, Vondrakova $\mathbf{D}^{5}$, Tomandl $\mathrm{J}^{6}$, Sevcikova $\mathrm{J}^{2}$, Lipkova $\mathrm{J}^{2}$, Benesova $\mathrm{K}^{7}$ and Jarkovsky $\mathbf{J}^{7}$

${ }^{1}$ Ist Internal Cardioangiological Department, St. Ann University Hospital, Faculty of Medicine, Masaryk University, Brno, Czech Republic

${ }^{2}$ Pathophysiologic Department, Faculty of Medicine, Masaryk University, Brno, Czech Republic

3 Internal Cardiologic Department, Brno University Hospital, Faculty of Medicine, Masaryk University, Brno, Czech Republic

${ }^{4}$ International Clinical Research Center (ICRC), St. Ann University Hospital, Brno, Czech Republic

${ }^{5}$ Cardiological Department, Hospital Homolka, Prague, Czech Republic

${ }^{6}$ Biochemistry Department, Faculty of Medicine Masaryk University, Brno, Czech Republic

${ }^{7}$ Statistical Department IBA, Masaryk University, Brno, Czech Republic

"Corresponding author: Monika Spinarova, Ist Internal Cardioangiological Department, St. Ann University Hospital, Faculty of Medicine, Masaryk University, Brno, Czech Republic, Tel: 00420723622747; E-mail: spinarova.monika@fnusa.cz

Received: May 24, 2018; Accepted: June 11, 2018; Published: June 13, 2018

Copyright: (c) 2018 Spinarova L, et al. This is an open-access article distributed under the terms of the creative commons attribution license, which permits unrestricted use, distribution, and reproduction in any medium, provided the original author and source are credited.

Abstract
The aim of the study is to evaluate the impact of new humoral substances: copeptin and mid- regional pro-
adrenomedullin (MR-proADM) on one-year survival of patients with stable systolic chronic heart failure (CHF) and to
compare them with AHEAD score assessing the comorbidities. The FAR NHL (FARmacology and NeuroHumoraL
activation) registry is a database of patients with stable CHF (ejection fraction (EF) $<50 \%$ ) treated in specialized HF
departments. AHEAD score is a simple bed-side mortality predictive model based on age and comorbidities. Primary
endpoint after 1-year follow-up was: death or hospitalization for decompensation of HF or heart transplantation or
LVAD implantation. To whole FAR NHL registry, a total amount of 1088 patients were included, in 552 of them the
levels of copeptin and MR-proADM were available. Mean age was $65+12$ years, mean EF was $31+9 \%$. Patients
without primary endpoint were assigned as group A ( 469 pts), those with the primary endpoint group B $(83$ pts).
There were statistically significant differences between the groups in the levels of copeptin: group A median 15.9
pmol/l (3.4-50.9) vs group B 23.7 pmol//(5.0-89.44) (p<0.001), MR-proADM: group A median 0.63 nmol//( $(0.32-1.34)$
vs group B 0.74 nmol/l (0.4-1.94) (p<0.001). Relationship of AHEAD score to primary endpoint in the first year of
follow-up was not significant, but within 24 th month it reached statistical significance: $p=0.017$. Patients with higher
AHEAD score (more comorbidities) reached more often the primary end-point. The cutt-off value $\geq 23.7$ for copeptin
had $50.6 \%$ sensitivity and $73.3 \%$. The cut - off value for MR-proADM $\geq 0.58$ had higher sensitivity $79.5 \%$ and lower
specificity $42.0 \%$. For both humoral substances there was statistical significant difference for discrimination of
patients with primary endpoint in lower AHEAD score groups. However, in the highest AHEAD score the level of
these substances lost their predictive value.

Keywords: Chronic heart failure; AHEAD score; Copeptin; Midregional pro-adrenomedullin; Prognosis

\section{Introduction}

Chronic heart failure is a clinical syndrome, which is best characterized by increased levels of various humoral substances, mostly the natriuretic peptides. Novel biomarkers, which could predict more accurate the outcome of patients with chronic heart failure are constantly under research.

Copeptin (also known as CT-proAVP) is a C-terminal fragment of pre-pro-vasopressin (Pre-proAVP). It is containing 39-amino acids. Pre-proAVP is a precursor protein which consists of a signal peptide, arginine vasopressin (AVP), neurophysin II and copeptin. Arginine vasopressin (AVP), also known as antidiuretic hormone, is one of the key hormones in maintaining osmolality and therefore in maintaining the volume of water in the extracellular fluid. It is synthesized mainly in the paraventricular neurons of the hypothalamus and in the supraoptical nucleus. Pre-pro-AVP is proteolytically cleaved into three fragments: vasopressin, neurophysin II and copeptin. It is well known, that the level of vasopressin correlates with the severity of heart failure. However, vasopressin is difficult to determine because of its short halflife and instability. On the contrary copeptin appears to have better stability and more simple detection, that provide it an alternative marker of AVP [1].

Adrenomedullin $(\mathrm{ADM})$ is a hormone primarily with vasodilatative effect in human body. It is derived from a larger molecule (preproADM) by posttranslational process. This peptide is divided into two inactive fragments (mid-regional part of proADM and the C-terminal fragment). ADM routine measurement is not suitable for clinical praxis, because of its low stability, while mid-regional proADM is stable and reliable surrogate for measurement marker of ADM release [2].

\section{Materials and Methods}

The FAR NHL (FARmacology and NeuroHumoraL activation) registry is a database of patients treated in departments with 
Citation: Spinarova L, Spinarova M, Goldbergova-Pavkova M, Spinar J, Parenica J, et al. (2018) Prognostic Impact of Copeptin and MidRegional Pro-Adrenomedullin in Chronic Heart Failure with Regard to Comorbidities. J Cardiovasc Dis Diagn 6: 326 . doi: $10.4172 / 2329-9517.1000326$

Page 2 of 5

specialized HF care in three University hospitals. Anamnestic data were prospectivelly collected from November 2014 till November 2015. The patients should have been treated for systolic heart failure: ejection fraction of left ventricle $(\mathrm{EF})<50 \%$ and stable for at least one month, follow up was one year.

Copeptin and mid-regional pro-adrenomedullin (MR-proADM) were evaluated: cut- points, sensitivity and specificity was calculated for each substance. The plasma samples were collected, directly frozen and stored in $-80^{\circ} \mathrm{C}$. Biomarker of cardiovascular load - MR-proADM - was detected using the homogeneous sandwich fluoroimmunoassay on the automate KRYPTOR (B.R.A.H.M.S AG, Hennigsdorf/Berlin, Germany). The B.R.A.H.M.S KRYPTOR ${ }^{\star}$ is a fully automated homogeneous random access platform. The system uses the Time Resolved Amplified Cryptate Emission (TRACE) technology [3].

The detection limit of the kit B.R.A.H.M.S MR-proADM KRYPTOR (Thermo Scientific) was $0.05 \mathrm{nmol} / \mathrm{L}$, the direct measurement range was $0.05 \mathrm{nmol} / \mathrm{L}-10 \mathrm{nmol} / \mathrm{L}$, enhanced with use of automatic dilution up to $100 \mathrm{nmol} / \mathrm{L}$. The functional assay sensitivity (inter-assay precision of $20 \% \mathrm{CV}$ ) has been assessed as being $0.25 \mathrm{nmol} / \mathrm{L}$. In concentration range of $>2 \mathrm{nmol} / \mathrm{L}-6 \mathrm{nmol} / \mathrm{L}$ was the intra-assay of $<2 \% \mathrm{CV}$, and the inter-assay of $\leq 10 \% \mathrm{CV}$.

The detection limit of the kit B.R.A.H.M.S Copeptin KRYPTOR (Thermo Scientific) was $0.69 \mathrm{pmol} / \mathrm{L}$, the direct measurement range was $0.7 \mathrm{pmol} / \mathrm{L}-500 \mathrm{pmol} / \mathrm{L}$, enhanced with use of automatic dilution up to $2000 \mathrm{pmol} / \mathrm{L}$. The functional assay sensitivity (inter-assay precision of $20 \% \mathrm{CV}$ ) has been assessed as being $1.08 \mathrm{pmol} / \mathrm{L}$. In concentration range of $4 \mathrm{pmol} / \mathrm{L}-15 \mathrm{pmol} / \mathrm{L}$ was the intra-assay of $<8 \%$ $\mathrm{CV}$, and the inter-assay of $\leq 10 \% \mathrm{CV}$.

Co-morbidities were evaluated by AHEAD score which was published in our previous studies. AHEAD score is a simple bed-side mortality predictive model for a wide population of AHF patients based on age and basic comorbidities. Based on multivariate analyses, previous published results, and clinical expert opinion, four parameters in addition to age were selected as follows: (A) atrial fibrillation, (H) haemoglobin level less than $130 \mathrm{~g} / \mathrm{l}$ for men and $120 \mathrm{~g} / \mathrm{l}$ for women, (E) elderly (age $\geq 70$ years), (A) abnormal renal parameters (creatinine $\geq 130 \mu \mathrm{mol} / \mathrm{l}$ ) and (D) diabetes mellitus. This model was used to divide patients according to long term survival, with a clear separation of Kaplan-Meier survival curves. An increase in the AUC value over time suggested that the long-term prognosis of patients, even that of heart failure patients, significantly depends on serious comorbidities [4].

Primary endpoint after 1-year follow-up was: death or hospitalization for decompensation of HF or heart transplantation (HTX) or LVAD implantation. This endpoint was related to the levels of copeptin and MR - proadrenomedullin.

The endpoint was caluculated for separate AHEAD scores. Patients signed an informed consent which was approved by Ethics committee and was in accordance with Helsinky declaration.

Standard descriptive statistics were applied in the analysis; absolute and relative frequencies for categorical variables and median supplemented with 5th-95th percentile range for continuous variables. Statistical significance of differences between groups of patients was computed using Fisher's exact test for continuous variables and MannWhitney $U$ test for continuous variables. The predictive power of variables for the mortality and their optimal cut-offs were analysed using ROC analysis and described by AUC, its confidence interval and statistical significance and cut-off sensitivity and specificity. The survival of patients was described and visualised using Kaplan-Meier methodology; statistical significance of differences in survival was analysed by log-rank test. The analysis was computed using SPSS 24.0.0.1 (IBM Corporation, 2016).

\section{Study population}

1088 patients were included into the FAR-NHL registry, 552 of them had the levels of copeptin and MR-proADM available. Mean age was 65 years, $81 \%$ were male, the etiology of $\mathrm{CHF}$ was ischemic heart disease in $53.5 \%$, dilated cardiomyopathy in $40.2 \%$ and $6.3 \%$ were classified as other. Mean EF was $31+9 \%$, mean systolic blood pressure was $128+16 \mathrm{mmHg}$ and diastolic blood pressure $80+10 \mathrm{mmHg}$, mean heart rate was $74+13 / \mathrm{min} .12 .9 \%$ of patients were classified as NYHA I, 67.2\% NYHA II and $19.9 \%$ as NYHA III and IV. Atrial fibrillation was presented in $35.0 \%$, diabetes mellitus type 2 in $35.1 \%$ and type 1 in $5.8 \%$.

\section{Result}

Patients without primary endpoint (death or hospitalization for decompensation of $\mathrm{HF}$ or heart transplantation or LVAD implantation) were assigned as group A (469 pts), those with the primary endpoint group B (83 pts).

There were statistically significant differences between the groups in the levels of copeptin: group A median $15.9 \mathrm{pmol} / \mathrm{l}$ (3.4-50.9) vs group B $23.7 \mathrm{pmol} / \mathrm{l}(4.8-89.4)(\mathrm{p}<0.001)$, MR-proADM: group A median $0.63 \mathrm{nmol} / \mathrm{l}(0.33-1.37)$ vs group B $0.76 \mathrm{nmol} / \mathrm{l}(0.34-1.94)(\mathrm{p}<0.001)$

Patients were then divided according to the AHEAD score into three groups: AHEAD score 0-1, AHEAD score 2-3, AHEAD score 4-5. Relationship of AHEAD score to primary endpoint in the first year of follow-up was not significant. Patients with higher AHEAD score (more comorbidities) reached more often the primary end-point (Table 1).

\begin{tabular}{|l|l|l|l|}
\hline AHEAD score & $\mathbf{N}$ & $\begin{array}{l}\text { Primary endpoint in the } \\
\text { 1styear }\end{array}$ & ${ }^{1} \mathbf{P}$ \\
\hline $0-1$ & 325 & $44(13.5 \%)$ & -- \\
\hline $2-3$ & 189 & $32(16.9 \%)$ & 0.424 \\
\hline $4-5$ & 38 & $7(18.4 \%)$ & -- \\
\hline All & 552 & $83(15.0 \%)$ & - \\
\hline${ }^{1}$ P-value of Fisher exact test. & & \\
\hline
\end{tabular}

Table 1: Relationship of AHEAD score and primary end-point in the first year of follow-up (death, hospitalization for AHF, HTX, LVAD).

In the extended follow-up, there was already statistical significance between the groups: AHEAD 0-1 vs. AHEAD 2-3 $\mathrm{p}=0,069$; AHEAD 0-1 vs. AHEAD 4-5 $\mathrm{p}=0,008$; AHEAD 2-3 vs. AHEAD 4-5 $\mathrm{p}=$ 0,118 , and $\log -$ rank test was $\mathrm{p}=0.017$ (Figure 1 ).

The cutt-off value $\geq 23.7 \mathrm{pmol} / \mathrm{l}$ for copeptin had $50.6 \%$ sensitivity and $73.3 \%$ specificity to predict primary outcome at the first year of follow -up, $\mathrm{p}<0.001$. The cut -off value for MR-proADM $\geq 0.57 \mathrm{nmol} / \mathrm{l}$ had higher sensitivity $79.5 \%$ and lower specificity $42.0 \%$ for one-year follow- up with $\mathrm{p}<0.001$. For both humoral substances there was statistical significant difference for discrimination of patients with 
Citation: Spinarova L, Spinarova M, Goldbergova-Pavkova M, Spinar J, Parenica J, et al. (2018) Prognostic Impact of Copeptin and MidRegional Pro-Adrenomedullin in Chronic Heart Failure with Regard to Comorbidities. J Cardiovasc Dis Diagn 6: 326. doi: $10.4172 / 2329-9517.1000326$

Page 3 of 5

primary endpoint in lower AHEAD score groups: copeptin $(\mathrm{p}<0.006$ and $\mathrm{p}<0.004)$, MR-proADM $(\mathrm{p}<0.008$ and $\mathrm{p}<0.002)$. However, in the highest AHEAD score the level of these substances lost their predictive value (Table 2).
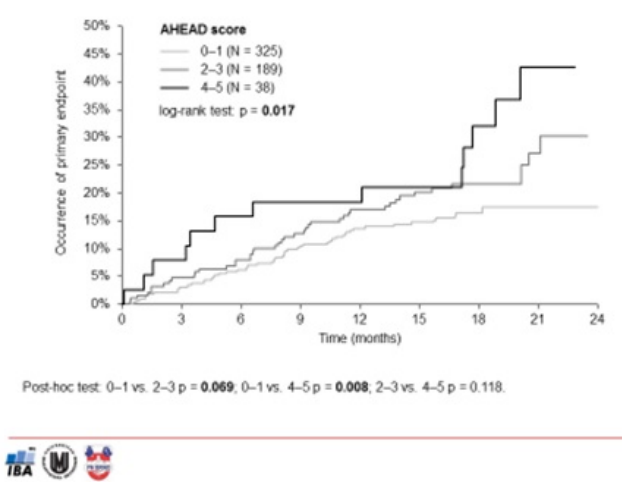

4 FAR NHL

Figure 1: Primary endpoint (death, hospitalization for AHF, HTX or LVAD) according to the basal AHEAD score.

\section{Discussion}

Vasopressin has been shown to increase with the severity of chronic HF. Copeptin is a fragment of pre-pro-vasopressin that is synthesized and secreted in equimolar amounts to vasopressin. Both hormones have a short lifetime in vivo, similar to BNPs, but in contrast to vasopressin, copeptin is very stable in vitro. The predictive value of copeptin has been shown in advanced HF, where it was superior to BNP for predicting 24-month mortality.

The study of Neuhold et al. evaluated the predictive value of copeptin in HF patients and compared it to B-type natriuretic peptide (BNP) and N-terminal pro-B-type natriuretic peptide (NT-proBNP). They reveal copeptins potential role as a single predictor of mortality in patients with NYHA II and III and in NYHA IV copeptin add additional information, but in this class the best predictive value was reveal by serum sodium. Higher level of copeptin was linked to excess mortality irrespective of clinical signs of heart failure. In this study copeptin appears to be superior to BNP or NT-proBNP, nevertheless these markers are closely related [5].

\begin{tabular}{|c|c|c|c|c|c|}
\hline Analysis & AUC (95\% IS) & ${ }^{1} \mathbf{P}$ & Cut-off & Sensitivity & Specificity \\
\hline \multicolumn{6}{|l|}{ All } \\
\hline Copeptin & $0.637(0.572 ; 0.703)$ & $<0.001$ & $\geq 23.7$ & $50.60 \%$ & $73.30 \%$ \\
\hline MR-proADM & $0.633(0.567 ; 0.699)$ & $<0.001$ & $\geq 0.58$ & $79.50 \%$ & $42.00 \%$ \\
\hline \multicolumn{6}{|c|}{ AHEAD score $0-1$} \\
\hline Copeptin & $0.629(0.542 ; 0.715)$ & 0.006 & $\geq 17.1$ & $59.10 \%$ & $66.20 \%$ \\
\hline MR-proADM & $0.624(0.532 ; 0,716)$ & 0.008 & $\geq 0.58$ & $68.20 \%$ & $60.90 \%$ \\
\hline \multicolumn{6}{|c|}{ AHEAD score 2-3 } \\
\hline Copeptin & $0.661(0.569 ; 0.774)$ & 0.004 & $\geq 26.0$ & $65.60 \%$ & $65.60 \%$ \\
\hline MR-proADM & $0.670(0.559 ; 0.781)$ & 0.003 & $\geq 0.87$ & $64.50 \%$ & $68.20 \%$ \\
\hline \multicolumn{6}{|c|}{ AHEAD score 4-5 } \\
\hline Copeptin & $0.576(0.340 ; 0.812)$ & 0.534 & $\geq 24.9$ & $85.70 \%$ & $41.90 \%$ \\
\hline MR-proADM & $0.525(0.283 ; 0.768)$ & 0.836 & $\geq 1.36$ & $57.10 \%$ & $61.30 \%$ \\
\hline
\end{tabular}

Table 2: ROC analysis of ability of copeptine and MR-proADM to predict primary outcome in the first year of follow-up.

In the study of Haehling et al. mid-regional pro-adrenomedullin levels increased with NYHA class and MR-proADM was a predictor of poor survival at 12 months $(\mathrm{p}=0.002)$. In receiver-operating characteristic curve analysis of 12-month survival, the area under the curve for MR-proADM and NT-proBNP was similar ( $\mathrm{p}=0.3$ ). Adding MR-proADM to the base model had stronger prognostic power than adding NT-proBNP (both p<0.01) [6].
In our study we evaluated the predictive value of copeptin and MRproADM over the comorbidities of patients with HF assessed by AHEAD score. Similarly like in citated article where copeptin was the more potent predictor of mortality in patients with NYHA II and III than NYHA IV, we found in our study that copeptine was more predictive for primary outcome (death, hospitalisation for AHF, HTX or LVAD) in patients with lower AHEAD score than with more comorbidities and higher AHEAD score. 
Citation: Spinarova L, Spinarova M, Goldbergova-Pavkova M, Spinar J, Parenica J, et al. (2018) Prognostic Impact of Copeptin and MidRegional Pro-Adrenomedullin in Chronic Heart Failure with Regard to Comorbidities. J Cardiovasc Dis Diagn 6: 326 . doi: $10.4172 / 2329-9517.1000326$

Page 4 of 5

Neuhold et al. have published a work concerning the comparison of copeptin and B-Type Natriuretic Peptide in NYHA IV patients. They proclaim that copeptin have a potential as a novel predictive marker in chronic systolic heart failure. The levels of this marker were inferior to sodium levels and especially GFR. They suspect, that hormones involved in fluid regulation supposed be of a limited value for prognosis because of renal function in patients with end-stage heart failure. The role of renal function as a marker of prognosis is well examined and the whole fluid-regulating hormone system connected with renal function might provide more specific information [5].

Repeated measurement of mid- regional pro-ANP and copeptin was assessed in the study of Miller. Biomarkers were collected every 3 months over 2 years and analysed in relation to death/transplantation. Combined increases in MR-proANP and copeptin $(p=0.03)$ with $c$ TnT $(p=0.02)$, and increases $\geq 30 \%$ above already raised values identified the patients at greatest risk A strategy of serial monitoring of MR-proANP and, of lesser impact, copeptin, combined with cTnT,may be advantageous in detecting and managing the highest-risk outpatients with HF [7].

The predictive role of copeptin and MR-proADM was also shown in The Biomarkers in Acute Heart Failure (BACH) trial. The highest levels of copeptin were predicitve for 90-days mortality and this was even more pronounced in patients with concomitant hyponatremia. Elevated levels of copeptin were bound with combined end points of mortality, readmissions, and emergency department visits [8].

Comparison of novel biomarkers were evaluated in several studies. Maisel et al. compared MR-proADM with B-type natriuretic peptide or troponin. MR-proADM was superior for predicting 90-day all-cause mortality in patients presenting with acute dyspnea $(\mathrm{p}<0.0001)$. Furthermore, MR-proADM added significantly to all clinical variables and it was also superior to all other biomarkers. MR-proADM was the biggest contributor to the predictive performance $[1,8]$.

Copeptin has correlations with already proven biomarkers, e.g. amino-terminal pro-B type natriuretic peptide (NT-proBNP) or cardiac troponin (hs-cTNT) as it was shown in the study of Tentzeris. In chronic stable heart failure patients, the correlations of copeptin were shown with hs-cTnT, creatinine, age and amino-terminal pro-B type natriuretic peptide (NT-proBNP) (all p<0.001). As well as hscTNT copeptin $(\mathrm{p}=0.002)$ was significantly increased in more severe patients with higher NYHA class. Copeptin was also important predictor of prognosis $(\mathrm{p}<0.001)$ [9].

Further study evaluated the capability B-type natriuretic peptide (BNP) as a prognostic marker in patients with acute destabilized heart failure in comparison with mid-regional pro-A-type natriuretic peptide (MR-proANP), mid-regional pro-adrenomedullin (MRproADM), and the C-terminal part of the arginine vasopressin prohormone (Copeptin). Increased BNP, MR-proANP, MR-proADM, and copeptin plasma level were proved as the strongest predictors of mortality in acute heart failure patients [10].

Novel biomarkers are thus promising in comparison with traditional natriuretic peptides and may bring a new additive information in prognosis of patients with heart failure.

Long term follow-up - five-year- were analysed in the study of Pozsonyi. Univariate analysis identified copeptin as a predictor of mortality. The final, multivariable Cox survival model identified a number of independent predictors of death. These included higher NYHA functional class, previous MI, at least one hospitalisation for worsening HF, elevated blood urea, NT-proBNP and copeptin levels and decreased GFR [11].

Our finding confirms these results and newly evaluates the relation of novel biomarkers with comorbidities.

There was even a meta-analysis from 17 prospective studies - nearly 6000 patients done by Zhang et al. A significant association was observed between circulating copeptin levels and risk of all-cause mortality in patients with HF. Copeptin levels were significantly higher in patients with HF who died during the follow-up period than in survivors [12].

\section{Conclusion}

Higher levels of new humoral substances: copeptin and MRproADM might identify HF patients with higher risk of adverse outcome. Our study is the first which evaluates the predictive value of copeptin and MR-proADM over the comorbidities of patients with HF assessed by AHEAD score.

We found that copeptine and MR-proADM were more predictive for primary outcome (death, hospitalisation for AHF, HTX or LVAD) in patients with lower AHEAD score than with more comorbidities and higher AHEAD score. Our finding newly evaluates the relation of novel biomarkers with comorbidities.

\section{Supporting information}

Supported by Ministry of Health, Czech Republic-conceptual development of research organization ( $\mathrm{FNBr}, 65269705)$.

\section{References}

1. Balling L, Gustafsson F (2014) Copeptin as a biomarker in heart failure. Biomarkers Med 8: 841-854.

2. Maisel A, Müller C, Nowak R, Peacock WF, Ponikowski P, et al. (2011) Midregion prohormone adrenomedullin and prognosis in patients presenting with acute dyspnea: Results from the BACH (Biomarkers in Acute Heart Failure) trial. JACC 58: 1057-1067.

3. Caruhel P, Mazier C, Kunde J, Morgenthaler NG, Darbouret B (2009) Homogeneous time-resolved fluoroimmunoassay for the measurement of midregional proadrenomedullin in plasma on the fully automated systém B.R.A.H.M.S KRYPTOR ${ }^{\circledR}$. Clin Biochem 42: 725-728.

4. Spinar J, Jarkovsky J, Spinarova L, Mebazzaa A, Gayat E, et al. (2016) AHEAD score-long-term risk clasification in acute heart failure. Int J Cardiol 202: 21-26.

5. Neuhold S, Huelsmann M, Strunk G, Stoiser B, Struck J, et al. (2008) Comparison of copeptin, B-type natriuretic peptide, and amino-terminal pro-B-type natriuretic peptide in patients with chronic heart failure: prediction of death at different stages of the disease. J Am Coll Cardiol 52: $266-272$

6. Von Haehling S, Filippatos GS, Papassotiriou J, Cicoira M, Jankowska EA, et al. (2010) Mid-regional pro-adrenomedullin as a novel predictor of mortality in patients with chronic heart failure. Eur J Heart Fail 12: 484-491.

7. Miller WL, Hartman KA, Grill DE, Struck J, Bergmann A, et al. (2012) Serial measurements of midregion proanp and copeptin in ambulatory patients with heart failure: Incremental prognostic value of novel biomarkers in heart failure. Heart 98: 389-394.

8. Maisel A, Xue Y, Shah K, Mueller C, Nowak R, et al. (2011) Increased 90day mortality in patients with acute heart failure with elevated copeptin: Secondary results from the Biomarkers in Acute Heart Failure (BACH study). Circ Heart Fail 4: 613-620. 
Citation: Spinarova L, Spinarova M, Goldbergova-Pavkova M, Spinar J, Parenica J, et al. (2018) Prognostic Impact of Copeptin and MidRegional Pro-Adrenomedullin in Chronic Heart Failure with Regard to Comorbidities. J Cardiovasc Dis Diagn 6: 326. doi: 10.4172/2329-9517.1000326

Page 5 of 5

9. Tentzeris I, Jarai J, Farhan S, Perkmann T, Schwarz MA, et al. (2011) Complementary role of copeptin and high-sensitivity troponin in predicting outcome in patients with stable chronic heart failure. Eur J Heart Fail 13: 726-733.

10. Gegenhuber A, Struck J, Dieplinger B, Poelz W, Pacher R, et al. (2007) Comparative evaluation of B-Type natriuretic peptide, mid-regional ProA-type natriuretic peptide, mid-regional pro-adrenomedullin, and copeptin to predict 1-Year mortality in patients with acute destabilized heart failure. J Car Fail 13: 42-49.
11. Pozsonyi Z, Förhécz Z, Gombos T, Karádi I, Jánoskuti L, et al. (2015) Copeptin (C-terminal pro Arginine-Vasopressin) is an independent longterm prognostic marker in heart failure with reduced ejection fraction. J Heart Lung Circ 24: 359-367.

12. Zhang P, Wu X, Li G, Sun H, Shi J et al. (2017) Prognostic role of copeptin with all-cause mortality after heart failure: A systematic review and metaanalysis. The Clin Risk Mang 13: 49-58. 